\title{
Pancreatitis por hipertrigliceridemia asociada a anticonceptivos orales: Reporte de un caso
}

\author{
Henry Altamar ${ }^{1}$, Ingrid Stephens ${ }^{2}$
}

${ }^{1}$ Residente de Endocrinología, Universidad del Rosario - Fundación Cardioinfantil, Bogotá, Colombia

${ }^{2}$ Médica Internista e Endocrinóloga, Fundación Santa Fe de Bogotá, Colombia

Fecha de recepción: 14/02/2017

Fecha de aceptación: 16/02/2017

\section{Resumen}

$\mathrm{L}$ a hipertrigliceridemia es una de las causas de pancreatitis aguda, generalmente con un riesgo mayor cuando su valor supera los $1.000 \mathrm{mg} / \mathrm{dl}$. Se han descrito diferentes alternativas terapéuticas para el tratamiento de pacientes con pancreatitis por hipertrigliceridemia, entre ellas la infusión de insulina. Se reporta el caso de un paciente con hipertrigliceridemia muy severa que presentó pancreatitis aguda y su evolución fue favorable con la terapia con insulina.

Palabras Clave: hipertrigliceridemia, pancreatitis, insulina, anticonceptivos.

\section{Introducción}

La hipertrigliceridemia es uno de los trastornos del metabolismo de lípidos más frecuentes, con una prevalencia en aumento por su relación con condiciones como obesidad, síndrome metabólico y diabetes. La hipertrigliceridemia da cuenta de $1 \%$ a $4 \%$ de las causas de pancreatitis aguda, mientras que la relacionada con medicamentos es responsable del $5 \%(1,2)$.

La hipertrigliceridemia es definida, según el Adult Treatment Panel III (ATP III), como niveles de triglicéridos en ayunas mayores a $150 \mathrm{mg} / \mathrm{dl}^{(3)}$. Sin embargo, los rangos de referencia pueden variar según la edad y raza, siendo más bajos en afroamericanos. En cuanto al género pueden ser más altos en los hombres ${ }^{(4)}$. Por su parte, la Asociación Americana de Endocrinología clasifica la hipertrigliceridemia en cuatro categorías, dos de ellas definidas como severa y muy severa con niveles de triglicéridos entre 1000-1999 mg/dl y mayor de $2000 \mathrm{mg} / \mathrm{dl}$, respectivamente, las cuales se relacionan con riesgo de desarrollar pancreatitis ${ }^{(5)}$. Tal riesgo es del $5 \%$ con niveles de triglicéridos mayores a $1000 \mathrm{mg} / \mathrm{dl}$ y del $10 \%$ al $20 \%$ para mayores a $2000 \mathrm{mg} / \mathrm{dl}^{(6)}$.
Se presenta el caso de una paciente con pancreatitis aguda secundaria a hipertrigliceridemia.

\section{Reporte del caso}

Mujer de 31 años de edad, sin antecedentes personales o familiares de dislipidemia a relacionar, en planificación con etinilestradiol 0,02 mg /Clormadinona acetato 2,0 mg. Consultó al servicio de urgencias por dolor abdominal generalizado, sin mejoría con antinflamatorios no esteroideos y sin otros síntomas asociados. Los estudios iniciales evidenciaron leve leucocitosis con neutrofilia, leve hiperbilirrubinemia indirecta y sin alteración en la amilasa. Se practicó ultrasonografía de abdomen que evidenció hígado graso sin otras alteraciones. Dada la persistencia del dolor abdominal se realizó una tomografía de abdomen que documentó pancreatitis aguda Baltazar D (figura 1). Como parte del estudio de la etiología de la pancreatitis se hizo un estudio del perfil lipídico que mostró: triglicéridos: $2.575 \mathrm{mg} / \mathrm{dl}$ y colesterol total: $489 \mathrm{mg} / \mathrm{dL}$. Se confirmó hipertrigliceridemia como causa de la pancreatitis en posible relación con la terapia anticonceptiva, dado que no se documentaron otros factores de riesgo. Se decidió traslado a unidad de cuidados intensivos para dar manejo con infusión de insulina, con lo cual se lograron niveles de triglicéridos inferiores a $500 \mathrm{mg} / \mathrm{dl}$ al sexto día de instaurado dicho manejo (figura 2). La paciente fue dada de alta, finalmente, con terapia combinada con fibrato y estatina.

Figura 1. Pancreatitis aguda Baltazar D

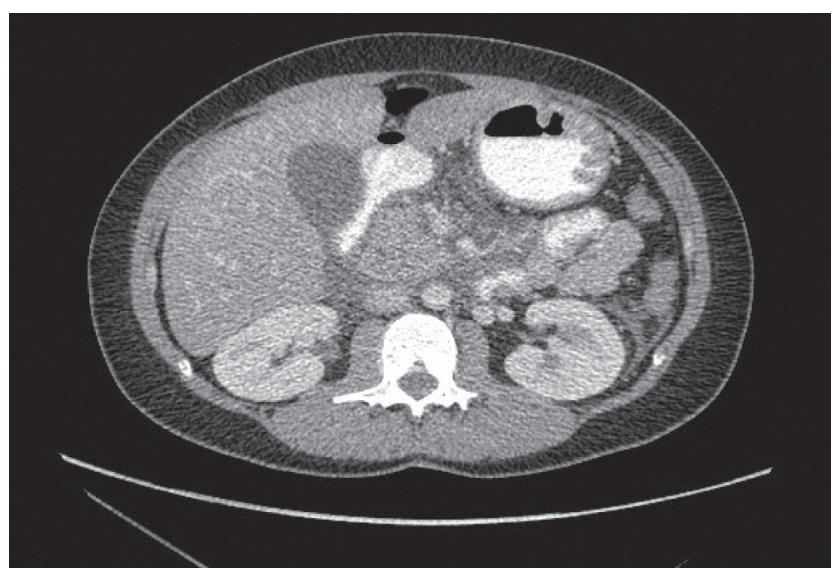


Figura 2. Comportamiento de los triglicéridos con la infusión de insulina

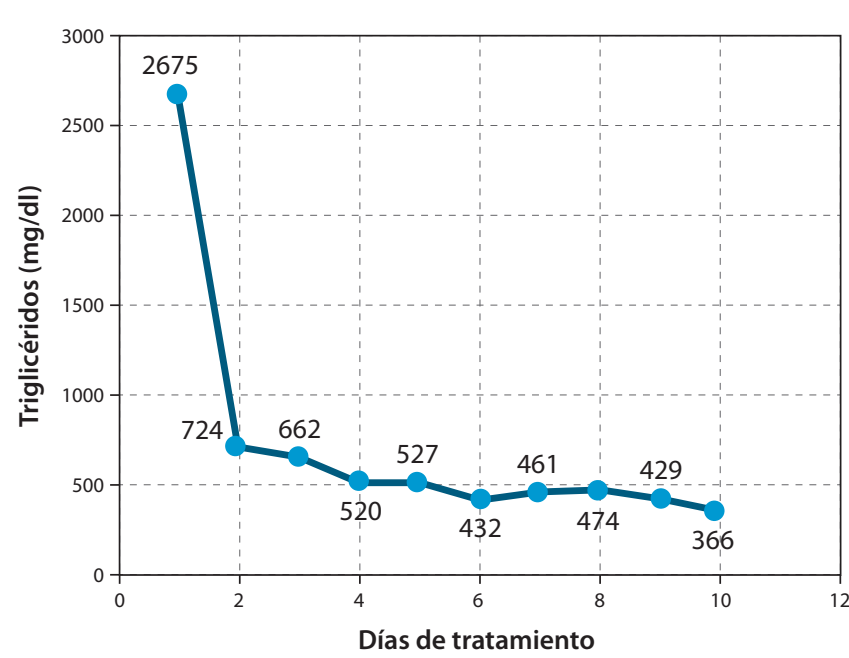

\section{Discusión}

En condiciones de ayuno de 14 horas, los triglicéridos son transportados en el plasma por las lipoproteínas de muy baja densidad (VLDL), por el contario, en estado posprandial son transportados por los quilomicrones. Tanto las VLDL como quilomicrones son hidrolizados por la lipoproteína lipasa (LPL). Tanto los trastornos heredados como los adquiridos del metabolismo de los triglicéridos han sido identificados como causa de hipertrigliceridemia.

Las hipertrigliceridemias pueden presentarse como el resultado de una alteración primaria del metabolismo lipídico por una causa subyacente de tipo genético, que se expresa por mecanismos de sobreproducción, disminución de la depuración o combinaciones de los anteriores. Por su parte, las de origen secundario incluyen como causa de mayor frecuencia las diabetes tipo 1 y 2 . Los niveles de triglicéridos se encuentran elevados en pacientes con pobre control glucémico o en el contexto de la cetoacidosis diabética. La prevalencia de diabetes oscila entre $42 \%$ y $72 \%$ en series de pacientes con pancreatitis por hipertrigliceridemia ${ }^{(7,8)}$. El mecanismo en la diabetes, así como en la obesidad está dado por resistencia a la insulina que conlleva a una mayor libración de ácidos grasos libres con mayor producción de triglicéridos.

El mecanismo exacto por el cual la hipertrigliceridemia causa pancreatitis no ha sido completamente dilucidado. Entre los mecanismos propuestos se encuentran el efecto tóxico directo de los ácidos grasos sobre el páncreas, la activación del tripsinógeno por los altos niveles de ácidos grasos libres y por defectos en la lipoproteína lipasa para hidrolizar los quilomicrones que pueden alterar la circulación pancreática, generando la respuesta inflamatoria ${ }^{(9)}$.
El alcohol disminuye la actividad de la lipoproteína lipasa, incrementa la producción hepática de VLDL y quilomicrones ${ }^{(10)}$, por lo cual el consumo pesado de alcohol también puede generar pancreatitis en pacientes con hipertrigliceridemia de base ${ }^{(11,12)}$.

Entre las causas medicamentosas se encuentran el clomifeno, los inhibidores de proteasa, el propofol, la olanzapina, los diuréticos tipo tiazidas y los betabloqueadores, entre otros $^{(13,14)}$. Los efectos de los estrógenos pueden ser evidentes durante el embarazo, anticoncepción y la terapia de remplazo hormonal. Los anticonceptivos orales combinados han sido relacionados con alteraciones en los marcadores hemostáticos, del metabolismo de lípidos y carbohidratos. Los estrógenos aumentan la producción de VLDL por parte del hígado, por lo cual no se recomienda la terapia hormonal en mujeres con triglicéridos mayores a $500 \mathrm{mg} / \mathrm{dl}$. El régimen combinado de etinilestradiol $0,02 \mathrm{mg}$ y clormadinona acetato $2,0 \mathrm{mg}$ puede incrementar los niveles de triglicéridos en un 68,8\% y el colesterol total en $12,1 \%{ }^{(15)}$. Lo anterior incrementaría el riesgo de pancreatitis por hipertrigliceridemia.

En los pacientes que desarrollan pancreatitis por hipertrigliceridemia el tratamiento es el convencional para pancreatitis aguda, incluyendo la reanimación hídrica, analgesia y el tratamiento de otras causas, con el objetivo de llevar los triglicéridos a una meta por debajo de $500 \mathrm{mg} / \mathrm{dl}$, lo cual ha demostrado conducir a mejoría clínica ${ }^{(16)}$. En las alternativas terapéuticas están descritos el uso de insulina, heparina y aféresis, entre otras.

La aféresis permite bajar los niveles de triglicéridos en un periodo corto de tiempo, lo cual puede ser beneficioso en casos severos o muy severos que comprometan la vida, asociados a hipocalcemia, acidosis láctica o disfunción orgánica. Mediante la aféresis se eliminan los triglicéridos disponibles en VLDL y quilomicrones del suero y se impide la generación de ácidos que causan efectos locales y sistémicos ${ }^{(17,18)}$. En una serie se documentó una reducción del $41 \%$ en los niveles de triglicéridos después de una sesión. Tras cada sesión, los niveles de triglicéridos deben ser revalorados y si están por debajo de $500 \mathrm{mg} / \mathrm{dl}$ se puede suspender la aféresis ${ }^{(19,20)}$.

La infusión de insulina en una unidad de cuidados intensivos es otra opción para el manejo de la pancreatitis relacionada con triglicéridos. Puede iniciarse en el rango de 0,1 a 0,3 unidades/hora con monitoreo glucémico por horario para ajustarla según los niveles de glucosa. En los pacientes sin hiperglucemia debe adicionarse dextrosa endovenosa para prevenir la hipoglucemia. Típicamente en pocos días, con un promedio de 3 a 4 días, se logra tener niveles de triglicéridos en la meta antes anotada, con lo cual se puede suspender la terapia. La insulina reduce la hipertrigliceridemia al mejorar la actividad de la lipoproteína lipasa que, a su vez, acelera el metabolismo de los quilomicrones y VLDL ${ }^{(21)}$.

El uso de la heparina está descrito en varias series y reportes de casos, basando su efecto sobre los triglicéridos en 
la estimulación de la liberación endotelial de la lipoproteína lipasa; sin embargo, su uso es controversial, dado que tal liberación es seguida por una mayor degradación hepática de la heparina, lo que conlleva a un agotamiento de los depósitos plasmáticos de la lipopotreína, lipasa y así al aumento en los niveles de quilomicrones y, por otra parte, se aumenta el riesgo de sangrado ${ }^{(22,23)}$.

Para la prevención de nuevos episodios de pancreatitis se requieren cambios en los estilos de vida que incluyen una dieta baja en grasas y carbohidratos simples, la abstinencia del alcohol, el control de los factores secundarios como la diabetes, y la interrupción de la medicación que esté implicada ${ }^{(24)}$. Los fibratos son los medicamentos de primera línea para el tratamiento de hipertrigliceridemia. Los inhibidores de la HMG-CoA reductasa tienen un efecto de disminución de los triglicéridos débil, y no deben usarse como monoterapia para hipertrigliceridemia; sin embargo, pueden tener un efecto reductor de lípidos sinérgico en combinación con fibratos y de- ben considerarse en pacientes en los que la hipertrigliceridemia severa no se controla solo con fibratos. En tal situación el fenofibrato no aumenta los niveles de estatinas, y se considera el agente más seguro para la terapia combinada ${ }^{(25)}$.

\section{Conclusión}

La pancreatitis por hipertrigliceridemia es una entidad asociada con morbimortalidad importante, dada su asociación con componentes del síndrome metabólico como la diabetes y en algunos casos con mal control glucémico. Ante la presentación de pancreatitis por hipertrigliceridemia y ausencia de diabetes o alteraciones primarias del metabolismo de los lípidos deben explorarse otras causas, como ingesta de alcohol o medicamentos que potencialmente eleven los triglicéridos. Entre las opciones terapéuticas, la infusión de insulina logra llevar los triglicéridos en pocos días a niveles menores de 500 $\mathrm{mg} / \mathrm{dL}$ ayudando así a la resolución de la pancreatitis y evitando sus complicaciones.

\section{Referencias}

1. Tsuang W, Navaneethan U, Ruiz L, Palascak JB, Gelrud A. Hypertriglyceridemic pancreatitis: presentation and management. Am J Gastroenterol. 2009;104(4):984-91.

2. Tenner S. Drug-induced acute pancreatitis: underdiagnosis and overdiagnosis. Dig Dis Sci. 2010;55(10):2706-8.

3. National Cholesterol Education Program (NCEP) Expert Panel on Detection Ea, and Treatment of High Blood Cholesterol in Adults (Adult Treatment Panel III). Third Report of the National Cholesterol Education Program (NCEP) Expert Panel on Detection, Evaluation, and Treatment of High Blood Cholesterol in Adults (Adult Treatment Panel III) final report. Circulation. 2002;106(25):3143-421.

4. Ford ES, Giles WH, Dietz WH. Prevalence of the metabolic syndrome among us adults: Findings from the third national health and nutrition examination survey. JAMA. 2002;287(3):356-9.

5. Berglund L, Brunzell JD, Goldberg AC, Goldberg IJ, Sacks F, Murad MH, et al. Evaluation and treatment of hypertriglyceridemia: an Endocrine Society clinical practice guideline. J Clin Endocrinol Metab. 2012;97(9):2969-89.

6. Scherer J, Singh VP, Pitchumoni CS, Yadav D. Issues in hypertriglyceridemic pancreatitis: an update. J Clin Gastroenterol. 2014;48(3):195-203.

7. Fortson MR, Freedman SN, Webster PD. Clinical assessment of hyperlipidemic pancreatitis. Am J Gastroenterol. 1995;90(12):2134-9.

8. Lloret Linares C, Pelletier AL, Czernichow S, Vergnaud AC, Bonnefont-Rousselot D, Levy P, et al. Acute pancreatitis in a cohort of 129 patients referred for severe hypertriglyceridemia. Pancreas. 2008;37(1):13-2.

9. Khan AS, Latif SU, Eloubeidi MA. Controversies in the etiologies of acute pancreatitis. JOP. 2010;11(6):545-52.

10. Brucker C, Hedon B, The HS, Höschen K, Binder N, Christoph A. Long-term efficacy and safety of a monophasic combined oral contraceptive containing $0.02 \mathrm{mg}$ ethinylestradiol and $2 \mathrm{mg}$ chlormadinone acetate administered in a 24/4-day regimen. Contraception. 2010;81(6):501-9.

11. Haber PS, Wilson JS, Apte MV, Hall W, Goumas K, Pirola RC. Lipid intolerance does not account for susceptibility to alcoholic and gallstone pancreatitis. Gastroenterology. 1994;106(3):742-8.

12. Goyal H, Smith B, Bayer C, Rutherford C, Shelnut D. Differences in Severity and Outcomes Between Hypertriglyceridemia and Alcohol-Induced Pancreatitis. N Am J Med Sci. 2016;8(2):82-7.

13. Arbel Y, Weinstein D, Yogev R, Halevy A. Acute pancreatitis following clomiphene citrate treatment: case report and review of the literature. Int J Surg. 2008;6(6):483-4.
14. Devlin JW, Lau AK, Tanios MA. Propofol-associated hypertriglyceridemia and pancreatitis in the intensive care unit: an analysis of frequency and risk factors. Pharmacotherapy. 2005;25(10):1348-52.

15. Winkler UH, Röhm P, Höschen K. An open-label, comparative study of the effects of a dose-reduced oral contraceptive containing $0.02 \mathrm{mg}$ ethinylestradiol/2 mg chlormadinone acetate on hemostatic parameters and lipid and carbohydrate metabolism variables. Contraception. 2010;81(5):391-400.

16. Toskes PP. Hyperlipidemic pancreatitis. Gastroenterol Clin North Am. 1990;19(4):783-91.

17. Yang F, Wang Y, Sternfeld L, Rodriguez JA, Ross C, Hayden MR, et al. The role of free fatty acids, pancreatic lipase and $\mathrm{Ca}+$ signalling in injury of isolated acinar cells and pancreatitis model in lipoprotein lipase-deficient mice. Acta Physiol (Oxf). 2009;195(1):13-28.

18. Szczepiorkowski ZM, Winters JL, Bandarenko N, Kim HC, Linenberger ML, Marques MB, et al. Guidelines on the use of therapeutic apheresis in clinical practice--evidence-based approach from the Apheresis Applications Committee of the American Society for Apheresis. J Clin Apher. 2010;25(3):83177.

19. Stefanutti C, Di Giacomo S, Labbadia G. Timing clinical events in the treatment of pancreatitis and hypertriglyceridemia with therapeutic plasmapheresis. Transfus Apher Sci. 2011;45(1):3-7.

20. Stefanutti C, Julius U. Treatment of primary hypertriglyceridemia states-General approach and the role of extracorporeal methods. Atheroscler Suppl. 2015;18:85-94.

21. Mikhail N, Trivedi K, Page C, Wali S, Cope D. Treatment of severe hypertriglyceridemia in nondiabetic patients with insulin. Am J Emerg Med. 2005;23(3):415-7.

22. Näsström B, Olivecrona G, Olivecrona T, Stegmayr BG. Lipoprotein lipase during continuous heparin infusion: tissue stores become partially depleted. J Lab Clin Med. 2001;138(3):206-13.

23. Jain P, Rai RR, Udawat H, Nijhawan S, Mathur A. Insulin and heparin in treatment of hypertriglyceridemia-induced pancreatitis. World J Gastroenterol. 2007;13(18):2642-3.

24. Ballantyne CM, Grundy SM, Oberman A, Kreisberg RA, Havel RJ, Frost PH, et al. Hyperlipidemia: diagnostic and therapeutic perspectives. J Clin Endocrinol Metab. 2000;85(6):2089-112.

25. Rosenson RS. Current overview of statin-induced myopathy. Am J Med. 2004;116(6):408-16. 